\title{
Alexithymia, Depression, Trait-anxiety and Their Relation to Self-reported Retrospective Dream Experience
}

\author{
Ornella Montebarocci*, Sara Giovagnoli \\ Department of Psychology, University of Bologna, Bologna, Italy \\ Email address: \\ ornella.montebarocci@unibo.it(O. Montebarocci), sara.giovagnoli@unibo.it (S. Giovagnoli) \\ ${ }^{*}$ Corresponding author
}

\section{To cite this article:}

Ornella Montebarocci, Sara Giovagnoli. Alexithymia, Depression, Trait-anxiety and Their Relation to Self-reported Retrospective Dream Experience. American Journal of Applied Psychology. Vol. 8, No. 6, 2019, pp. 121-132. doi: 10.11648/j.ajap.20190806.13

Received: November 14, 2019; Accepted: December 2, 2019; Published: December 7, 2019

\begin{abstract}
The present research explored the relationship between alexithymia, self-reported depression and trait anxiety and dreaming experience in a non-clinical sample. Characteristics of the alexithymia trait, such as the deficit in the symbolic representation of emotions and the limited imaginative ability, can have an impact on the richness and quality of dream experience. Depression and trait anxiety are both reported to be related to alexithymia and dreaming. A group of 30 non-clinical subjects (20 females) participated in the study. Alexithymia was assessed with the Toronto Structured Interview for Alexithymia (TSIA), an observer-report instrument composed of 24 questions that assesses a general subjective level of alexithymia and 4 dimensions of alexithymia: Identifying emotional feelings (DIF), Describing emotional feelings (DDF), Imaginal Processes (IMP); and Externally oriented thinking (EOT). The subjective dreaming experience was assessed with the Mannheim Dream Questionnaire (MADRE), an instrument that was designed to elicit some form of dream history including dream recall frequency, use and correlates of dream, attitude towards dream and presence of different kinds of dreams experience, such as nightmares, lucid dreams, and déjà-vu. The BDI-2 and the STAIY2 were administered to evaluate negative affect. Two multivariate multiple regression analysis were carried on. Results showed that alexithymia was related to dream correlates and to attitude towards dream. Trait anxiety was found to be related to nightmares frequency and self-reported depression to déjà-vu event. In the future, it would be interesting to use a larger and more representative sample. The use of clinical population, such as nightmare sufferers or patients with sleep and/or mental disorders, could possibly disclosure further meaningful relationships among affect dysregulation, affect modulation and self-reported retrospective dream characteristics.
\end{abstract}

Keywords: Alexithymia, Depression, Trait Anxiety, Dreams Correlates, Attitude Towards Dreams

\section{Introduction}

Alexithymia is a construct which comprises a pragmatic and externally oriented cognitive style that leads to a difficulty in recognizing and distinguishing between feelings and bodily sensations and in describing feelings to others [1]. A restricted imagination, marked by the paucity of fantasies, dreams, and daydreaming along with a poor imaginative aptitude and a diminished affect-related fantasy, are key features of alexithymia [2-4].

Early clinical impressions have signposted that alexithymia might be especially associated with an impoverished dream experience and have linked an alexithymic response style with a diminished dream recall frequency and with a lower degree of vividness or complexity in dreaming $[5,6]$. Clinicians also described that alexithymic patients rarely or never report nocturnal dreams or, when requested, to recount their dreams as literal and concrete, with boring and unhelpful associations. In fact, even if few studies have not found any relationships between alexithymia and the emotional content of dreams [7], the dreams of alexithymic people are often reported to be lacking in fantasy, bizarreness and symbolism. [8-10].

Some studies have reported evidence that specific sensory and structural features in dreams are altered among alexithymic patients [11] and, in particular, a decreased imaginative involvement and an externally-oriented thinking style in alexithymia have been consistently documented in 
daydreaming, fantasy, and nocturnal dreams [9, 10, 12]. Very similar physiognomies have been reported for clinical samples with alexithimic characteristics, such as skin disordered patients [13], asthmatics [14, 15] and sleep disordered [16], and among people with alexithimic features in the general population [8-10, 17].

Recently, Nielsen and colleagues [12] formally suggested that people who suffer from alexithymia and people with alexithymic characteristics presented, together with a lack of introspection and of the symbolic function, a dreaming component disorder due to the deficit of the mentalization process. Such a failure interferes with the production of complex and elaborate dream material and hampers the recall of dreams and dream associations.

Besides, although recognizing that alexithymic people's dreams may be minimally recalled or barren and bland, Taylor and colleagues [18] proposed that alexithymic individuals may also have dreams with unregulated primary or latent content, such as bizarre, emotionally intense, and disturbing dreams, including nightmares. Indeed, the same paradoxical combination of both impoverished and nightmarish dreams has been reported by other authors. In specific, some studies found that individuals with alexithymia, along with a lack of interest for their own personal dreams and inability to identify a personal meaning, also experience frequent nightmares and distressing dreams [8, 12]. Undeniably, nightmares as dreams may reflect failures in emotion regulation [15] and the deficit in the emotion regulation of alexithymia may account for the association between alexithymia and nightmare.

On the other hand, nightmares appear also associated with negative affect. In fact, empirical evidence has basically demonstrated that individuals who have frequent nightmares report that major distressing life events frequently precipitate their nightmares and several authors suggested that negative dreams and recurrent nightmares were also frequently reported in depressive patients and are associated with mood disorder, depressive symptoms, and "negative attitude towards the self" $[19,20]$. Moreover, since nightmares production is also related to a personality style characterized by intense reactive emotional distress and anxiety [21], many studies have investigated the association between nightmare frequency and various measures of anxiety, indicating a weak to moderate relationship between anxiety and retrospective estimates of nightmare frequency [22, 23].

In sum, considering the essential role of dreams in ensuring an affective adaptation and regulation, more research is needed to clarify relationships among alexithymic factors, self-reported depression, anxiety and various attributes of dreams, including nightmares.

Moreover, although converging evidence indicates that dream impoverishment and altered dreams experience is associated both with clinical and non-clinical alexithymia and with self-assessed depression and trait anxiety, it is important to notice that results regarding dream frequency (including nightmare frequency) and dream content differ depending on which methods are used for collecting data - retrospective questionnaires or prospective dream diariesand no explicit interpretation can be, so far, suggested about all the mentioned relations.

In the present study, the 24-item Toronto Structured Interview for Alexithymia [TSIA; 24] was used to assess alexithymia. The TSIA is a reliable structured interviewbased method that, as an external rated device, prevents the bound of a self-evaluative approach as for example, do the dominant measures for assessing alexithymia [25, 26]. This instrument has been recently proved to have no stable connection to self-reported negative affect [27] and, an additional strength of the TSIA, is to introduce items for detecting the lack of imaginative and fantasy aspects of the alexithymia construct $[28,29]$. The TSIA, in fact, comprises a fourth factor labeled Imaginal processes (IMP), besides the three classical factors named Difficulty in identifying feelings (DIF), Difficulty in describing feelings (DDF), and Externally oriented thinking (EOT) [30]. The scores on the IMP scale of the TSIA were particularly attractive, for the purpose of the present research, given that individual imaginal aptitude appears to be relevant in dreaming activity [8].

The Mannheim Dream Questionnaire [MADRE; 31, 32] was instead administered to measure dream-related aspects. The MADRE is a valid multidimensional, retrospective, selfreport questionnaire developed to assess a dream comprehensive individual history and several components of dreaming such as the dream recall frequency, the use and correlates of dream, the attitude towards dream, and, finally, the presence of different types of dreams, such as nightmares, lucid dreams and déjà vu.

The Spielberger Trait-Anxiety Inventory-Y2 [STAI-Y2; $33,34]$ and the Beck Depression Inventory-II [BDI-II; 35, 36] were both used to assess the presence of trait anxiety and depressive symptoms.

A first general aim of the present study was to assess whether the broad construct of alexithymia, its dimensions, self-assessed depression and trait anxiety, could be possibly related to independent aspects of self-reported, retrospective dreams experience in a non-clinical sample.

The reviewed literature led us to hypothesize that different components of dreaming - dream recall, individual attitude towards dream, use and correlates of dreams, nightmares, in particular-would be differently predicted by TSIA total score and by the TSIA subscale scores, as well as by the BDI-II and the STAI-Y2 scores.

Especially, as previously stated, although some research reported an association between alexithymia and nightmares $[8,37]$, consistent amount of studies have also demonstrated that frequent nightmares are associated with a number of personality traits such as heightened imagery involvement, fantasy proneness, psychological absorption, depression and anxiety [21]. Considering such an assumption, more than alexithymia, we would expect that negative affect, could be possibly predictive of specific altered types of dreams, such as nightmares, lucid-dreams or even déjà vu experience. 


\section{Method}

\subsection{Participants}

Ethical approval for the study was obtained by the Ethical Committee of the University of Bologna. All participants gave their written informed consent before inclusion in the study and were debriefed at the end of the experimental session.

A total of 30 participants ( 20 women), recruited in several Italian university departments and enrolled from the general population through personal contacts, have participated in the study. The mean age for the total sample was 26.7 years (age range $=17$ to 45 years; $\mathrm{SD}=6.6$ ) with a mean age of 29.6 years $(\mathrm{SD}=7.21)$ and of 25.3 years $(\mathrm{SD}=6.0)$ for men and women, respectively. No significant difference in the mean age of participants in relation to their gender was found $\left(\mathrm{t}{ }_{(28)}\right.$ $=-1.7 ; \mathrm{p}=.09)$. Overall, most were singles $(83.3 \%)$ and employed (63.3\%). The educational level was high $(86.7 \%$ of the participants have got high school diplomas or above). 2 subjects have declared to usually use medical remediation such as psychotropic drugs or painkillers and 10 subjects have declared to use often drugs or alcohol. Finally, 6 subjects have reported having recently suffered from emotional stress (mourning, work overload, financial stress...). No significant gender differences were found for education $\left(\chi 2_{(1)}=.14, \mathrm{p}=.70\right)$, marital status $\left(\chi 2_{(1)}=.12\right.$, $\mathrm{p}=.73)$, occupation status $\left(\chi 2_{(2)}=.86, \mathrm{p}=.65\right)$. The sample was homogeneous for gender also in drugs/alcohol use $\left(\chi 2_{(1)}=.30\right.$, $\mathrm{p}=.58)$, for medical remediation use $\left(\chi 2_{(1)}=.27, \mathrm{p}=.61\right)$ and for emotional distress $\left(\chi 2_{(1)}=.94, p=.33\right)$. Descriptive statistics for sociodemographic characteristics and general clinical-medical information were collected through a brief questionnaire in a categorical scheme that only included two levels (yes/no format). Descriptive statistics of the whole sample are presented in Table 1.

Table 1. Frequencies and percentages of the total sample $(n=30)$ according to Participant's Sex, Marital Status, Education Level, Drugs/alcohol use, Medical remediation use, Emotional Stress experience.

\begin{tabular}{llll}
\hline & & Frequency & Percentage \\
\hline \multirow{2}{*}{ Gender } & Males & 10 & 33.3 \\
& Females & 20 & 66.7 \\
Marital status & Singles & 25 & 83.3 \\
& Married/Cohabitating & 30 & 16.7 \\
& Separated/widowed & - & - \\
Occupation & Employed & 16 & 53.3 \\
& Student & 12 & 6.7 \\
Education level & Unemployed/retired & 2 & 40.0 \\
Drugs and & High-Level Education & 26 & 13.3 \\
alcohol & Yes & 10 & 86.7 \\
Psychotropic & No & 20 & 33.3 \\
drugs & Yes & 2 & 66.7 \\
Emotional Stress & No & 28 & 6.7 \\
& Nos & 6 & 93.3 \\
& No & 24 & 20.0 \\
\hline
\end{tabular}

\subsection{Measures and Procedure}

Participants were administered with the Italian version of the Toronto Structured Interview for

Alexithymia [TSIA; 24] and they also completed the Mannheim Dream Questionnaire [MADRE; 31, 32]. A backtranslation procedure was adopted for the MADRE. The Italian form of the MADRE was translated into the Italian language by two English language experts. Then the Italian form obtained from the translations was translated back again into English by two other specialists in English language. The final translated form was compared with the original form and the consistency between them was confirmed by the authors.

Participants were also administered with the Italian version of the Spielberger State-Trait Anxiety Inventory-Y2 [STAIY2; 33, 34] and the Italian version of the Beck Depression Inventory-II [BDI-II; 35, 36] for assessing the current level of trait anxiety and depression symptoms.

The TSIA is composed of 24 interview items, six items for each of the four facets of the alexithymia construct - DIF (Difficulty in Identifying Feelings), DDF (Difficulty in Describing Feelings), EOT (External Orientation Thinking), and IMP (Mental Imaginal Processes). Each interview question includes a set of prompts and/or probes, keyed to the thematic content of the item, to elicit information assisting in the accuracy of the scoring. All items are scored on a three-point scale ranging from 0 to 2 with some scores based on the frequency of the presence of a characteristic, and others on the degree of the presence of the characteristic. Good psychometric property of the scale is reported by several authors $[25,26]$. Samples of TSIA items are: Are you sometimes puzzled of confused about what emotion you are feeling (DIF)?; Is it usually easy for you to find words to describe your feelings to others (DDF)?; Do you tend just to let things happen rather than trying to understand why they turn out a certain way (EOT)?; Is it rare for you to fantasize (IMP)? Two clinical researchers were first requested to become familiar with the concept of alexithymia, then they were also trained in the administration and scoring procedures for the TSIA [24]. The first interviewer transcribed verbatim the responses to the questions and the probes and then scored the 24 questions of the TSIA during each interview. The 30 interviews were all recorded to be used for the assessment of interrater reliability. The second independent evaluator scored $10 \%(n=3)$ out of the interviews. After reading a short instruction, and following the Informed Consent, the interview was completed on an individual basis. It has been taken about 35 minutes for each interview.

The MADRE is a 20 items self-report retrospective questionnaire, mixed measured in Likert scale instrument, that assesses basic characteristics of dream-related variables, such as the frequency of the recollection of dreams, the individual's attitudes towards dreams, - the personal meaning of one's own dreams and the impression that dreams provide impulses or pointers for waking life, - the use and correlates of dreams, - the effects that dream have on waking life, the frequency of dream sharing, the recording of dreams, the dreams affecting day-time mood, the creative 
dreams, the problem-solving dreams — and the frequency of different kind of dreams, - nightmares, lucid dreams and déjà vu- . As reported by Schredl and colleagues [32], for the nightmare items, a definition for nightmares based on the ICSD-3 [38] was used: "Nightmares are dreams with strong negative emotions that result in awakening from the dreams. The dream plot can be recalled very vividly upon awakening." Similarly, a definition adopted from Schredl and Erlacher [39] was presented for the lucid dreaming item: "In a lucid dream, one is aware that one is dreaming during the dream. Thus, it is possible to wake up deliberately, or to influence the action of the dream actively, or to observe the course of the dream passively." Finally, for déjà-vu was adopted the definition used in Funkhouser [40] and Neppe [41] "During a déjà-vu experience one is convinced one is reliving real-life situation that was already experienced in a dream". Examples of questions assessed within the MADRE are: "How often do you discuss your dreams with family and friends? How much attention to do you usually pay towards your dreams? In general, how many dreams do you remember per week?".

Recall of dreams, Nightmares frequency, Lucid dreaming frequency and Déjà-vu experiences were all considered in the analysis as separate factors.

Recall of dreams (item1) was assessed in a 7-point scale format $(0=$ never and $6=$ almost every morning. Actual nightmare frequency along with Lucid dreaming frequency and Déjà-vu experiences based on dreams (items 4, 10 and 18 , respectively) were assessed with an eight-point scale $(0=$ never and $8=$ several times a week).

Factor Attitude towards dreams, in terms of the personal meaning of one's own dreams and the impression that dreams provide impulses or pointers for waking life, was measured by item 12 which consists of eight sentences with a five-point format $(0=$ Not at all $/ 4=$ Totally $)$.

Most of the dream variables eliciting utilizations of dreams, such as frequency of dream sharing, recording of dreams, dreams affecting day-time mood, creative dreams, problem-solving dreams were in 8-points format with $0=$ never and $8=$ several times a week. (items 13-14-15-1617). In the present research, these items were summarized to obtain a new Factor labeled Correlates of dreams experience.

The BDI-II is a widely used 21-item self-report inventory that measures the current presence and degree of depression. High scores indicate severe depressive symptoms. Each question refers to a symptom related to depression and has a set of four possible answer choices, ranging in intensity.

The STAI-Y2 is a well-known anxiety self-evaluation questionnaire including 40 items, with a Likert scale from 1 to 4, grouped into two different 20-item forms: one measuring trait anxiety and the second measuring state anxiety scale. In this study, only the form assessing trait anxiety was used.

After compiling the brief clinical information sheet, the participants completed the TSIA Interview and the self-report questionnaires. The laboratory session lasted about one hour per participant.

\subsection{Statistical Analyses}

The data were analyzed by means of SPSS statistical package version 25 .

To evaluate the psychometrics property of all the different instruments used, Means, standard errors, Cronbach's Alphas, Mean Inter-items correlation (to evaluate the internal consistency reliability) have been considered. Moreover, the Interviewer-to-rater intraclass correlation reliability (ICC) estimates have been calculated for the TSIA Interview total scores and all its subscales, considering the score obtained by 2 different raters.

To exclude that the TSIA could be possibly associated with measures of negative affect, a Pearson correlation analysis has been used to evaluate the relation between Total score of the TSIA Interview, its DIF, DDF, EOT, IMP subscales and the BDI-II and STAI-Y2 scores.

Two different Multivariate multiple regression Analysis were conducted by means of the General Linear Model (GLM) procedure.

A first Multivariate multiple regression analysis was carried on evaluating if specific parameters of the TSIA Interview would be predictive of different dimensions of the MADRE questionnaire. In this analysis the TSIA Interview Total score and the subject's difficulty in identifying feelings (DIF), the difficulty in describing feelings (DDF), the external oriented thinking (EOT), the mental imaginal processes (IMP) were all considered as independent factors and the Attitudes toward dreams scores, the dreams' Correlates scores, the Dream recall frequency, the Lucid dreaming, Nightmare and Déjà-vu experience frequencies of the MADRE, were used as dependent variables.

A second Multivariate multiple regression analysis was conducted to evaluate if BDI-II and STAI-Y2 total scores could be predictive of the same different aspects measured by the MADRE questionnaire. In this second analysis, the total score of BDI-II and the STAI-Y2 were used as independent factors and the Attitudes toward dreams and the dreams' Correlates scores along with the Dream recall frequency, the Lucid dream frequency, the Nightmare frequency and Déjàvu experience frequency, were again considered as dependent variables.

\section{Results}

\subsection{Mean Scores, Reliability Estimates of all Scales and Interrater of the TSIA}

Means and standard errors of all the scales and subscales considered in the present study have been reported, for the total sample, in table 2. Means Inter-items Correlation (MIC) and Cronbach's alpha coefficients were used to evaluate the internal consistency reliability. The values obtained for MIC were included in the $0.15-0.50$ recommended range [42]; Cronbach's Alpha for DDF and total score of TSIA, as well as for STAY-Y2 and Correlates and Attitudes of the MADRE questionnaire exceeded the 0.70 criterion $[42,43]$. Although the DIF, the EOT and the IMP scales of the TSIA Interview 
and the BDI-II score showed lower Alpha indices than those usually recommended, however, as indicated by Cortina [44], the MIC is a better estimate of homogeneity for scales with a relatively few items than is the Cronbach's Alpha. In fact, the MICs for the DIF, DDF, EOT and IMP of the TSIA Interview and the BDI-II total score were higher than 0.15 , suggesting an adequate internal consistency. In sum, the different scales used in the present study met the standards of an adequate internal consistency (see table 2).

Table 2. Means, SEs, Cronbach's Alphas, Mean Inter-items Correlation (MIC; Means and variance) for the TSIA total and for the different subscales of the TSIA Interview, the BDI-II total score, the STAI-Y2 total score and the MADRE different facets. The Interviewer-to-rater intraclass correlation reliability (ICC) estimates for TSIA Interview are also reported.

\begin{tabular}{|c|c|c|c|c|c|}
\hline & & Mean \pm SE & Cronbach's Alpha & MIC (mean \pm variance) & ICC \\
\hline \multirow{5}{*}{ TSIA } & $D I F$ & $5.03 \pm 2.88$ & 0.67 & $0.26 \pm 0.34$ & $.929 *$ \\
\hline & $D D F$ & $5.53 \pm 3.45$ & 0.78 & $0.38 \pm 0.01$ & .889 \\
\hline & $E O T$ & $6.20 \pm 3.17$ & 0.69 & $0.27 \pm 0.05$ & $.980 *$ \\
\hline & $I M P$ & $7.10 \pm 2.48$ & 0.60 & $0.20 \pm 0.04$ & $.974 *$ \\
\hline & Total & $24.93 \pm 9.20$ & 0.86 & $0.21 \pm 0.04$ & $.959 *$ \\
\hline BDI-II & Total & $11.77 \pm 8.15$ & 0.64 & $0.16 \pm 0.05$ & - \\
\hline STAY-Y2 & Total & $43.77 \pm 10.01$ & 0.90 & $0.33 \pm 0.03$ & - \\
\hline \multirow{6}{*}{ MADRE } & Attitudes & $28.00 \pm 8.25$ & 0.94 & $0.68 \pm 0.01$ & - \\
\hline & Correlates & $24.60 \pm 6.92$ & 0.75 & $0.37 \pm 0.02$ & - \\
\hline & Recall & $3.67 \pm 1.90$ & - & - & - \\
\hline & Nightmares & $3.20 \pm 1.77$ & - & - & - \\
\hline & Déjà vu & $2.70 \pm 2.34$ & - & - & - \\
\hline & Lucid dreams & $3.43 \pm 1.89$ & - & - & - \\
\hline
\end{tabular}

Note: DIF = difficulty in identifying feelings facet scale; DDF = difficulty in describing feelings facet scale;

$\mathrm{EOT}=$ externally oriented thinking facet scale; IMP = imaginal processes facet scale; Total=Tsia total score

$* \mathrm{p}<0.05$.

Estimates of interrater reliability were calculated for the TSIA and its facet scales by examining the correspondence between scores rated by two independent expert-raters for the $10 \%$ of the total interviews' sample $(n=3)$. The intraclass correlation coefficient was used to assess the level of agreement [45]. The results were reported in table 2. The reliability estimates for the TSIA and the facet scales were all statistically significant for all the domains except for the DDF facet scale where the intraclass correlation coefficient was not statistically significant. However, all these estimates exceeded the 0.60 standards recommended by Landis and Koch [46], indicating an adequate interrater reliability.

\subsection{Correlation Between TSIA Interview, Its Subscales and Negative Affect}

Table 3. Pearson correlation analysis between TSIA total and for the different subscales of the TSIA Interview, BDI-II total score and STAI-Y2 total score.

\begin{tabular}{llll}
\hline & & STAY-Y2 & BDI-II \\
\hline \multirow{4}{*}{ Tsia } & DIF & $\mathrm{r}=.31$ & $\mathrm{r}=.16$ \\
& DDF & $\mathrm{r}=.12$ & $\mathrm{r}=.15$ \\
& EOT & $\mathrm{r}=-.08$ & $\mathrm{r}=-.10$ \\
& IPM & $\mathrm{r}=-.20$ & $\mathrm{r}=.06$ \\
Total & $\mathrm{r}=.02$ & $\mathrm{r}=.08$ \\
\hline
\end{tabular}

Note: $* \mathrm{p}<.05 ; * * \mathrm{p}<.001$

To exclude that the TSIA Interview (specifically created to measure the presence of alexithymia trait), could be an implicit measures of negative affectivity, a Pearson correlation analysis was used to control for the relation between the different facets of the TSIA Interview (Total score, DIF, DDF, EOT, IMP) and the BDI-II and STAI-Y2 total scores. No significant correlation has been found between TSIA Total and the different scales of the TSIA Interview, the BDI-II total score and the STAI-Y2 total score (see table 3).

\subsection{Multivariate Multiple Regression Analysis}

The results from the first Multivariate multiple regression analysis, showed that the different parameter of the TSIA Interview were not significantly predictive as general effect of the dream's characteristics measured by the MADRE questionnaire (DIF: Wilks' Lambda $=0.803, \mathrm{~F}_{6,18}=0.736, \mathrm{p}=.63$; Partial Eta Square $=0.197$; DDF: Wilks' Lambda $=0.688$, $\mathrm{F}_{6,18}=1.362, \mathrm{p}=.28$; Partial Eta Square=0.312; EOT: Wilks' Lambda $=0.662, \mathrm{~F}_{6,18}=1.535, \mathrm{p}=.22$; Partial Eta Square=0.338; IMP: Wilks' Lambda $=0.566, \mathrm{~F}_{6,18}=2.299, \mathrm{p}=.08$; Partial Eta Square $=0.434$; Total score: Wilks' Lambda $=0.687, \mathrm{~F}_{6,18}=1.365$, $\mathrm{p}=.28$; Partial Eta Square=0.313).

However, looking at the univariate analysis, it has been observed that specific subscales of the TSIA were predictive of specific aspects of the dream's characteristics. In particular, the TSIA total score was a significant predictor of the dream's correlates of the MADRE (see table 4). This result may suggest that to a higher presence of alexithymia matches a higher level of dream's correlates. The DDF, EOT and IMP scales of the TSIA resulted all significantly predictive of the dream's Correlates of the MADRE (see table 4). The regression parameters suggested that the subject's Difficulty in describing feelings (DDF), the External oriented thinking (EOT) and the Mental imaginal processes (IMP) were all negatively linked to the dream's Correlates of the MADRE (see figure 1, graph a, b, c). 

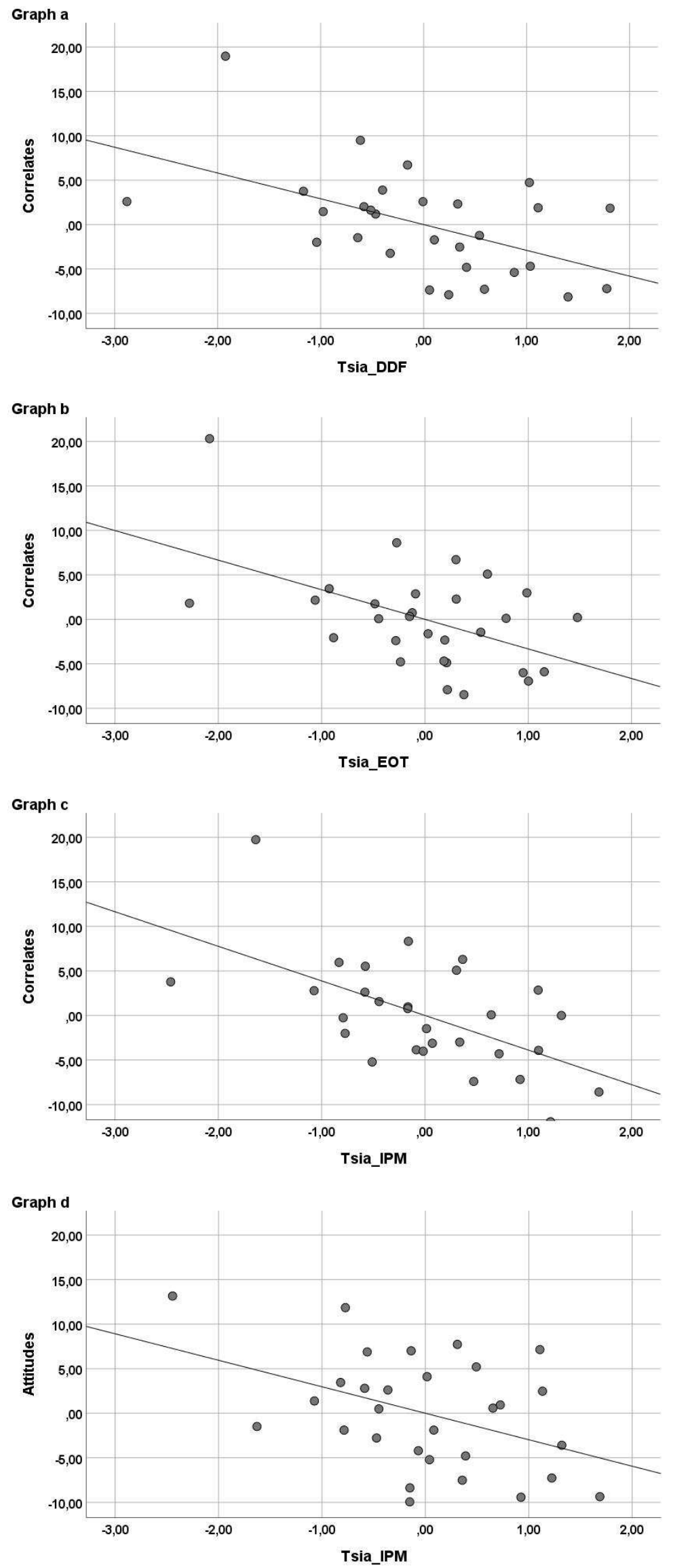

Figure 1. Scatterplots (partial regression plots) representing the negative relation between the DDF (a), the EOT (b) and the IMP (c) scales of the TSIA and the Dream's correlates of the MADRE, the graph d shows the negative relation between the IMP scale of the TSIA and the Attitudes toward dreams of the MADRE. 
A significant negative association was also found between the IMP scale of the TSIA and the Attitudes toward dreams of the MADRE, suggesting that the degree of imaginably of the subject could be a significant predictive factor of the individual degree of subject's Attitudes toward dreams of the MADRE (see figure 1, graph d).

The External oriented thinking (EOT) of the TSIA was a significant predictor of the frequency of déjà-vu experience of the MADRE. A low EOT score was related to a high frequency of déjà-vu experience of the MADRE (see figure 2 , graph b).

As reported in table 4, no significant relations have been found between the different scores calculated from the TSIA Interview and the dream recall frequency, the nightmares frequency and the lucid dreaming frequency of the MADRE questionnaire.

Table 4. Regression parameters related to the multivariate multiple regression analysis between the TSIA total score and its subscales and the different dimensions of the MADRE questionnaire.

\begin{tabular}{|c|c|c|c|c|c|c|}
\hline \multicolumn{7}{|l|}{ TSIA } \\
\hline & & DIF & $D D F$ & $E O T$ & $I M P$ & Total \\
\hline & & B (SE); t & B (SE); t & B (SE); t & B (SE); t & $\mathrm{B}(\mathrm{SE}) ; \mathrm{t}$ \\
\hline \multirow{4}{*}{ MADRE } & Attitudes & $\mathrm{B}=.61(.96) ; \mathrm{t}=.64$ & $\mathrm{~B}=-.43(1.14) ; \mathrm{t}=-.37$ & $\mathrm{~B}=-.76(1.38) ; \mathrm{t}=-.55$ & $\mathrm{~B}=-2.95(1.30) ; \mathrm{t}=-2.3^{*}$ & $\mathrm{~B}=.51(1.08) ; \mathrm{t}=.47$ \\
\hline & Dream Recall & $\mathrm{B}=-.17(.29) ; \mathrm{t}=-.60$ & $\mathrm{~B}=-.61(.34) ; \mathrm{t}=-1.79$ & $\mathrm{~B}=-.34(.42) ; \mathrm{t}=-.81$ & $\mathrm{~B}=-.69(.39) ; \mathrm{t}=-1.79$ & $\mathrm{~B}=.46(.33) ; \mathrm{t}=1.41$ \\
\hline & Nightmares & $\mathrm{B}=.29(.25) ; \mathrm{t}=1.14$ & $\mathrm{~B}=-.17(.30) ; \mathrm{t}=-.57$ & $\mathrm{~B}=-.16(.37) ; \mathrm{t}=-.44$ & $\mathrm{~B}=-.34(.35) ; \mathrm{t}=-.99$ & $\mathrm{~B}=.08(.29) ; \mathrm{t}=.29$ \\
\hline & Déjà-vu experience & $\mathrm{B}=-.26(.26) ; \mathrm{t}=-1.02$ & $\mathrm{~B}=-.41(.31) ; \mathrm{t}=-1.34$ & $\mathrm{~B}=-.88(.37) ; \mathrm{t}=-2.35^{*}$ & $\mathrm{~B}=-.65(.35) ; \mathrm{t}=-1.85$ & $\mathrm{~B}=.52(.29) ; \mathrm{t}=1.80$ \\
\hline
\end{tabular}

Note: $\mathrm{B}=$ standardized $\mathrm{b} ; \mathrm{SE}=$ Standard Error; DIF = difficulty in identifying feelings facet scale; DDF = difficulty in describing feelings facet scale; EOT = externally oriented thinking facet scale; IMP = imaginal processes facet scale; Total=Tsia total score. ${ }^{*} \mathrm{p}<.05 ;{ }^{*} \mathrm{p}<.001$

The results from the second Multivariate multiple regression analysis, showed that the BDI-II questionnaire was significantly related to the dimensions measured by the MADRE questionnaire $\left(\mathrm{F}_{6,21}=3.16, \mathrm{p}=.023\right.$, Partial Eta Squared =.475). In particular, the results suggested a significant and positive relation between the BDI-II total score and the Déjà-vu frequency of the MADRE (see table 5; figure 2, graph a). As reported in table 5, no other significant relations have been found between the BDI-II score and all the dreams variables of the MADRE considered.
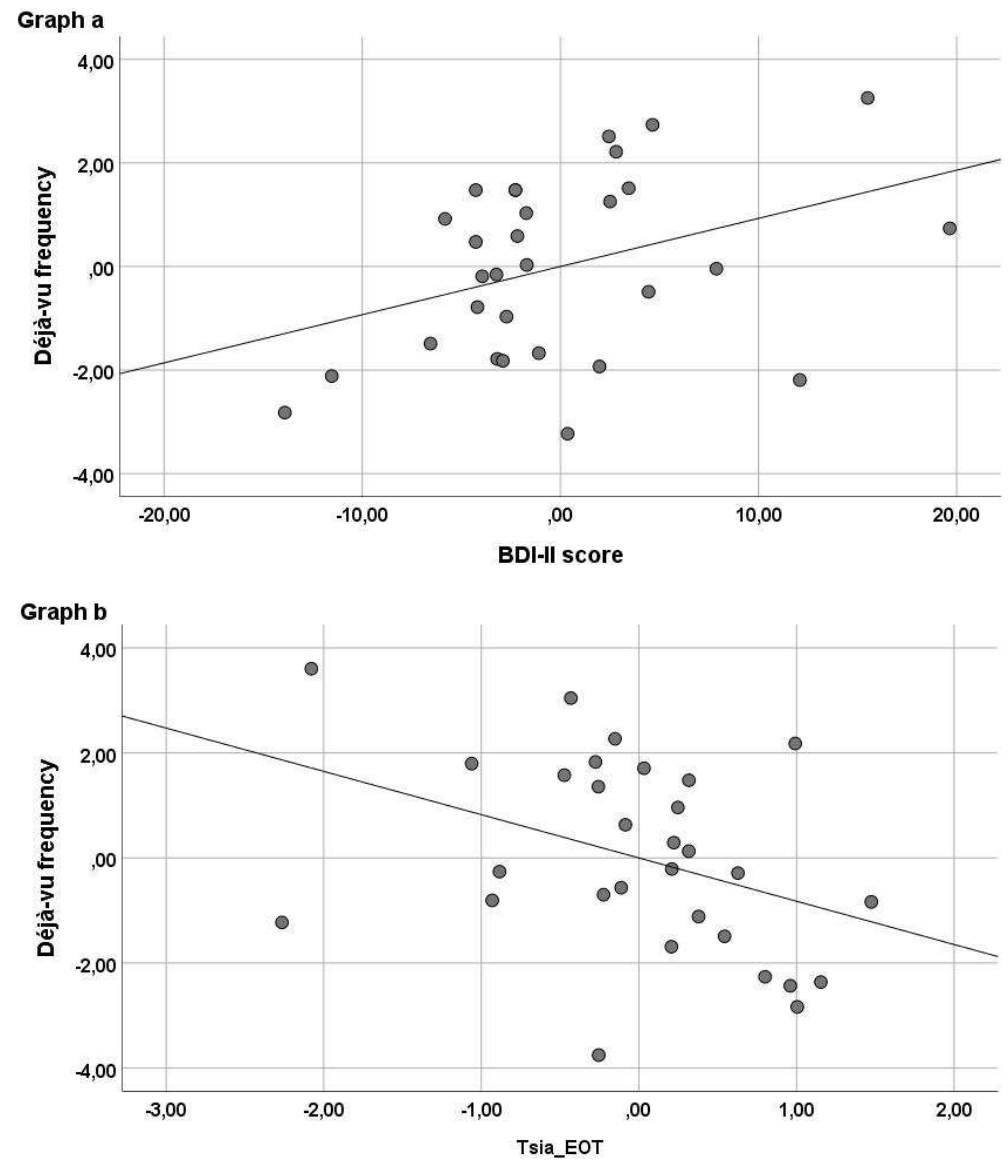

Figure 2. Scatterplot (partial regression plots) representing the positive relation between BDI-II score and Déjà-vu experience of the MADRE (a) and the negative relation between EOT scale of the TSIA and the Déjà-vu experience of the MADRE (b). 
The STAI-Y2 questionnaire was significantly related to the dreams dimensions of the MADRE considered $\left(\mathrm{F}_{6,21}=\right.$ 2.72, $\mathrm{p}=.041$, Partial Eta Squared =.438). In particular, the results showed a significant and positive relation between the STAI-Y2 score and the Nightmare frequency (see table 5). The STAI-Y2 score resulted a significant predictor of the Nightmare frequency of the MADRE, suggesting that the counterpart of a higher level of trait-anxiety, was a greater number of nightmares (see figure 3). As reported in table 5, no more significant associations were found between the STAI-Y2 and the other dimensions of the MADRE.

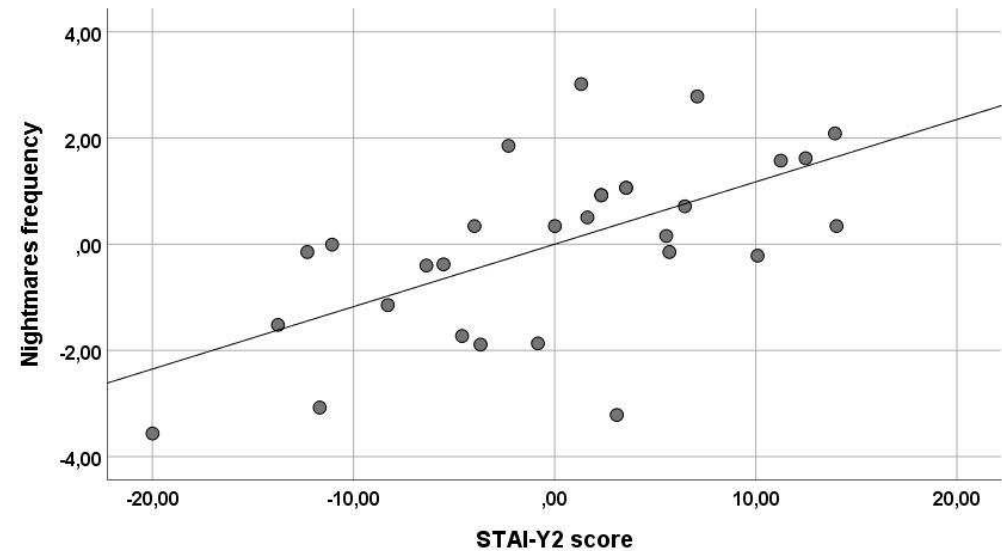

Figure 3. Scatterplot (Partial regression plot) representing the positive relation between the STAI-Y2 score and the frequency of the nightmares of the MADRE.

Table 5. Regression parameters related to the multivariate multiple regression analysis between the STAI-Y2, the BDI-II and the different dimensions of the MADRE questionnaire.

\begin{tabular}{|c|c|c|c|}
\hline & & STAY-Y2 & BDI-II \\
\hline & & $\mathrm{B}(\mathrm{SE}) ; \mathrm{t}$ & B (SE); $t$ \\
\hline \multirow{5}{*}{ MADRE } & Attitudes & $\mathrm{B}=-.34(.17) ; \mathrm{t}=-1.96$ & $\mathrm{~B}=-.25(.21) ; \mathrm{t}=-1.19$ \\
\hline & Correlates & $\mathrm{B}=.26(.14) ; \mathrm{t}=1.93$ & $\mathrm{~B}=-.02(.17) ; \mathrm{t}=-.10$ \\
\hline & Dream Recall & $\mathrm{B}=.011(.04) ; \mathrm{t}=.27$ & $\mathrm{~B}=.04(.05) ; \mathrm{t}=.87$ \\
\hline & Nightmares & $\mathrm{B}=.12(.03) ; \mathrm{t}=4.17 * *$ & $\mathrm{~B}=-.00(.04) ; \mathrm{t}=-.09$ \\
\hline & Déjà-vu experience & $\mathrm{B}=.03(.03) ; \mathrm{t}=.96$ & $\mathrm{~B}=.09(.04) ; \mathrm{t}=2.27^{*}$ \\
\hline
\end{tabular}

Note: $\mathrm{B}=$ standardized $\mathrm{b} ; \mathrm{SE}=\mathrm{Standard}$ Error; $* \mathrm{p}<.05 ; * \mathrm{p}<.001$

\section{Discussion}

The present research was designed to explore the relationship between alexithymia, its dimensions, presence of self-assessed depression and trait anxiety, and different kind of self-reported dream experiences.

Characteristics of the alexithymia trait, such as the deficit in the symbolic representation of emotions together with limited imaginative ability, can have an impact on the recall of dreams, richness or quality of dreams production, their meaning and experience.

A group of non-clinical subjects was administered with the TSIA to assess alexithymia and the MADRE to evaluate different aspects of dreams. The first, is a valid interviewbased measure that wholly evaluate the full range of features of the alexithymia construct including a new factor named the reduced fantasy/constricted fantasy dimension. The second, is a reliable, self-reported, retrospective, mixed measured instrument for assessing basic characteristics of dream-related variables. Participants also compiled the BDI-
II and the STAI-Y2 to control for the presence of depression and trait anxiety.

Results from the present study, confirmed good psychometric property for the TSIA, the BDI-II, the STAIY2 and the MADRE. Consistent to data reported in recent literature [27] the TSIA interview showed no association with both self-reported depression and trait anxiety, as no significant correlations have been found between TSIA total and all its facets and scores on BDI-II and STAI-Y2. The TSIA also exhibited excellent interrater reliability estimates, both comparable in magnitude to those reported in the current literature [24, 26].

Results from the present study, confirmed that alexithymia, self-assessed depression and trait anxiety, reveal several meaningful independent relationships with different characteristics of dreaming, such as their use and correlates, the individual attitude towards personal's own dreams and different kind of dreams experiences. Of all the dream variables considered, merely the dream recall dimension, did not prove to be significantly predicted by either alexithymia in general and its four dimensions nor depression and trait anxiety. Self-assessed depression and trait anxiety were 
significant predictors of Déjà-vu and Nightmare frequency, respectively.

Interestingly, of all the scales of the TSIA, three were predictive of different dreams factors: the emotional component of alexithymia focused on the difficulty in describing feelings (DDF scale) and the cognitive components centered on the use of a concrete and poorly introspective way of thinking (EOT and IMP scales). The DDF, the EOT and the IMP scales of the TSIA were all negatively correlated to participant's Correlates of dream of the MADRE and the IMP scale was also negatively linked to the Attitude towards dreams of the MADRE. That is, the typical deficit in describing feelings, the concrete way of thinking and the specific symbolic deficit of individuals with alexithymic trait, were all negatively linked with the importance that the subject attributes to dreams and with the quality of the elaboration and use of dreams. These results seem coherent with those reported by several authors which reveal that people with alexithymic characteristics, in nonclinical population, rated their dreams as boring and not interesting and were found to not believing in the importance, meaning and usefulness of their dreams $[8,9]$.

The TSIA total score was instead found positively associated to the scale Correlates of dreams of the MADRE. That is, the more is the score on alexithymia, the higher is the dream's use and sharing. This result appears, in some way, counterintuitive and we presume that it may be due to the low number of participants or to the set of populations participating. It is also plausible to remember that the MADRE, as a retrospective dream questionnaire, measures respondents' evaluations about dream experiences in general and not the real or actual dream experiences [47]. This consideration might account for the result obtained. Certainly, more studies are needed to clarify the implications of this result.

No significant association has been found, in the present data, among alexithymia and all its facets, selfassessed depression, trait anxiety and Dream recall frequency. The lack of significant associations appears to suggest that, both dysregulation of affect and negative modulation of mood seem not related to the number of recollected dreams. Many researches have reported discrepant findings, with some authors finding a positive association $[9,10,12]$ while others not [23]. This puzzling picture may be explained by incongruities in the procedure of collecting dream reports and by the representativeness of subject dream production [8]. Therefore, further research is needed, before offering a definite interpretation. In fact, such a result, for the present sample, may be due to the use of a relatively small, non-clinical sample or to the use of a retrospective tool in dreaming experience recollection. This may possibly have allowed an underestimation of dreams frequency.

Regarding self-reported depression and trait anxiety, in the present study, trait anxiety was found to be predictive of Nightmares frequency. In contrast, Alexithymia did not predict the frequency of nightmares, nor any of its

\section{dimensions.}

Although these results seem not aligned with those reported by part of the literature $[8,12,18,37]$ which stated that alexithymia in non-clinical samples was associated with dreams that are unregulated and nightmarish, data from the present study are entirely coherent with those of several authors which proved that bad dreams and nightmare production is frequently reported to have a high comorbidity with mood and anxiety disorders [15, 20, 23, 48].

In our opinion, although alexithymia could be an expression of an emotional regulation deficit, it also comprehends a remarkable shortfall in the symbolic access. Dreams could have a basic regulative and adaptive function, but the symbolizing activity of the imagination remains the central force in every dream. Since the access to dream experience is related to the symbolic potential of the individual, it is conceivable to suppose that alexithymia could possibly have an impact on dreaming activity, as the results of the present study revealed. Besides, nightmares represent a paradigmatic expression of dreams, extremely powerful and forceful, that needs an extra-symbolic work. This extra-elaboration comprises an elective access to personal meaningful experiences and an emotional expression and awareness. It is plausible to realize that this mentalization function that typifies nightmares, may be partially inaccessible for people who usually score highly on alexithymia.

Finally, depression and alexithymia were both significant predictors of Déjà-vu, but in opposite directions. In fact, the present study indicates that déjà vu experiences were reported very often and were related to self-reported depressive symptoms. On the contrary, the EOT of the TSIA was negatively linked to déjà vu experiences.

Although, empirical research on the déjà vu experience is scanty and the results have been far from conclusive [49], this result may be read as interesting. In fact, a study on déjà vu experiences and depersonalization in a sample of students reported that emotional sensitivity, unstable mood fluctuations, apathetic episodes and irregular working rhythm emerged as predisposing personality traits for déjà vu experience [50]. Results of another study of Wolfradt [51] with non-clinical population, showed that the most influential personality dimensions that were associated with déjà vu experiences were thin boundaries and selfabsorption and that déjà vu experiences were more closely related to daydreams. That is, persons with high selfabsorption or more tuned in to their mental experiences, with mood instability and altered work rhythms, as they could actually be depressive people, presumably experience the déjà vu event more intensely than persons with a concrete and literal way of approaching reality, as the alexithymics presumably do. Interestingly, since déjà vu can be thought of as a metacognitive competence [52], and the externally oriented thinking scale (EOT) represents the cognitive deficit of the alexithymia construct, then the negative association between the déjà vu and the EOT of the TSIA may be defendable. 
However, since studies reported no relations between déjà $\mathrm{vu}$ and negative affect [53], and no researches have been done, so far, on the relation between alexithymia and déjà vu, as suggested by Funkhouser and Schredl [54], additional research is highly desirable on déjà vu experiences, also via prospective measures, such as for example, diary keeping, and through studying whether the variables associated with déjà vu frequency also explain inter-individual differences in laboratory experiments.

In sum, results from the present study seem to be promising and interesting, but some limitations must be mentioned.

First, as we stated above, the sample size was small, and many correlations may be possibly have remained nonsignificant. A larger and more representative sample is clearly desirable in future studies. Moreover, it could be appropriate to use different clinic or psychiatric samples such as, for example, nightmare sufferers or patients with sleep and/or different mental disorders. Conceivably, studying the relationships between alexithymia and dreaming with a sample containing more highly alexithymic participants will yield to stronger and meaningful deductions.

Second, as we previously stated, the use of retrospective measures of dreaming may be a methodological weakness. Some dreaming attributes (e.g dream recall, nightmare frequency) may be seriously underestimated. In fact, for example, studies investigating the prevalence of nightmares have generally found higher frequencies in studies using daily logbooks or diaries compared to retrospective reports, raising the question as to whether retrospective questionnaires lead to an underestimation of nightmare frequency, possibly due to the suppression of the frightening nightmare experience $[31,55]$. In future studies, an assessment of group differences with several types of dreaming measurements, instead of just one retrospective multi-item dreaming questionnaire, is thus clearly desirable. In addition, although dreams recorded from spontaneous home awakenings are most relevant to the clinical setting, studies in which dream reports are obtained in sleep laboratories are strongly recommended. This would produce a better control over the condition of assessment, thus reducing unwanted variance in a participant's motivation and timing of dream reports.

\section{Conclusion}

Present results are overall promising and have confirmed the relevance of a significant association between alexithymia, self-reported depression, trait anxiety and different aspects of dreaming.

In line with previous studies, the difficulty in describing emotions, a concrete way of thinking and a low fantasy proneness are all predictive of a lower involvement in dreaming. It is worth to notice, that the use of the TSIA in assessing alexithymia, has proved to be useful due to the presence of the IMP scale. This imaginative scale, was a significant predictor of both Attitudes towards dream and
Correlates of dream, revealing an interesting association between alexithymia and dream experience. Thus, it remains unclear how exactly alexithymia as a broad dimension, is related to dreams Correlates.

On the contrary, nightmare and déjà vu experiences, seem to be better predicted by self-reported depression and trait anxiety, respectively.

However, more researches need to be implemented with a larger and more representative sample. The use of clinical could possibly disclosure further meaningful relationships among affect dysregulation, affect modulation and dreams characteristics. In addition, dreams and nightmares, are challenging to assess in a reliable way with the unique use of retrospective measures. Since these measures can be affected by factors like poor memory as well as by how the question is formulated. In future, it could be useful, to assess dreams variables in a combined way, such as for example, with daily dream log or in laboratory sessions.

Finally, the present study has also important clinical implications. Given that individuals with alexithymics characteristics seem to have problems in dealing with the abstract elaboration of emotions and dreams, it might be possible to modulate the symbolic deficit in alexithymia working on the dreaming components of people with a high incidence of alexithymic characteristics or in clinical populations frequently reported to be associated to substantial level of alexithymia. This can possibly facilitate diagnosis, therapeutic treatment and long-term therapeutic outcomes in alexithymia.

\section{Acknowledgements}

Assistance in collecting and analyzing data provided by Valentina Moscatiello was greatly appreciated by the authors.

\section{References}

[1] Taylor G. J., Bagby, M. R., and Parker, J. D. A., "Disorders of Affect Regulation: Alexithymia in Medical and Psychiatric Illness", 1999, Cambridge: Cambridge University Press.

[2] Caretti, V., La Barbera, D., and Capraro, G., "La Toronto Alexithymia Scale (TAS-20) ", in Alessitimia, valutazione e trattamento, Vincenzo Caretti, V., and La Barbera, D., Casa Editrice Astrolabio - Ubaldini Editore, Roma, 2005, pp. 17-23.

[3] Taylor, G. J., Bagby, R. M., and Parker, J. D. A., "I disturbi della regolazione affettiva", in L'alessitimia nelle malattie mediche e psichiatriche (Tr. it., 2000), 2007, Roma: Fioriti.

[4] Taylor, G. J., Bagby, R. M, and Parker, J. D. A. (2016). What's in the name "alexithymia"? A commentary on "Affective agnosia: Expansion of the alexithymia construct and a new opportunity to integrate and extend Freud's legacy. Neuroscience and Biobehavioral Reviews, 68, 1006-1020. doi.org/10.1016/j.neubiorev.2016.05.025.

[5] Apfel, R. J., and Sifneos, P. E. (1979). Alexithymia: Concept and measurement. Psychotherapy and Psychosomatics, 32, 180-190. 
[6] Krystal, H. (1979). Alexithymia and psychotherapy. American Journal of Psychotherapy, 33, 17-31.

[7] De Gennaro, L., Ferrara, M., Cristiani, R., Curcio, G., Martiradonna, V., and Bertini, M., (2003). Alexithymia and dream recall upon spontaneous morning awakening. Psychosomatic Medicine, 65, 301-306.

[8] Warnes, H. (1986). Alexithymia, clinical and therapeutic aspects. Psychotherapy and Psychosomatics, 46, 96-104.

[9] Lumley, M. A., and Bazydlo, R. A. (2000). The relationship of alexithymia characteristics to dreaming. Journal of Psychosomatic Research, 48, 561-567.

[10] Parker, J. D. A., Bauermann, T. M., and Smith, C. T. (2000). Alexithymia and impoverished dream content: Evidence from rapid eye movement sleep awakenings. Psychosomatic Medicine, 62, 486-491.

[11] Parker, J. D., Taylor, G. J., and Bagby, R. M. (2003). The 20Item Toronto Alexithymia Scale. III. Reliability and factorial validity in a community population. Journal of Psychosomatic Research 55, 269-275.

[12] Nielsen, T. A., Taylor, G. J., and Bagby, J. (2011). Dreaming correlates of alexithymia among sleep-disordered patients. Dreaming, 1, 1-16.

[13] Tantam, D., Kalucy, R., and Brown, D. G. (1982). Sleep, scratching and dreams in eczema: A new approach to alexithymia. Psychotherapy and Psychosomatics 37, 26-35.

[14] Monday, J., Montplaisir, J., and Malo, J. L. (1987). Dream process in asthmatic subjects with nocturnal attacks. American Journal of Psychiatry, 144, 638-640.

[15] Nielsen, T., and Levin, R. (2007). Nightmares, Bad Dreams, and Emotion Dysregulation: A Review and New Neurocognitive Model of Dreaming. Current Directions in Psychological Science, 18 (2), 84-88.

[16] Quellet, L., Nielsen, T. A., Montplaisir, J., Cartier, A., Malo, J. L., and Lassonde, M. (1996). L'alexithymie, re'ponse affective et re`ves: Investigation en laboratoire de trois caracte'ristiques sous-jacentesaude'ficitdel'expressiondese'motions. Revue Internationale De Psychopathologie, 23, 491-503.

[17] Bazydlo, R., Lumley, M. A., and Roehrs, T. (2001). Alexithymia and polysomnographic measures of sleep in healthy adults. Psychosomatic Medicine, 63, 56-61.

[18] Taylor, G. J., Parker, J. D. A., Bagby, R. M., and Bourke, M. P. (1996). Relationships between alexithymia and psychological characteristics associated with eating disorders. Journal of Psychosomatic Research 41, 561-568. doi: 10.1016/S0022-3999 (96) 00224-3.

[19] Beauchemin K. M., and Hays, P. J. (1996). Dreaming away depression: the role of REM sleep and dreaming in affective disorders. Affective Disorders, 41, 125-1.

[20] Sandman, N., Valli, K., Kronholm, E., Revonsuo, A., Laatikainen T., and Tiina Paunio, T. (2015). Nightmares: Risk Factors Among the Finnish General Adult Population. Sleep, 1, 38 (4), 507-14. doi: 10.5665/sleep.4560.

[21] Levin, R., and Nielsen, T. A. (2007). Disturbed dreaming, posttraumatic stress disorder, and affect distress: A review and neurocognitive model. Psychological Bulletin, 133, $482-528$.
[22] Levin, R., and Hurvich, M. S. (1995). Nightmares and annihilation anxiety. Psychoanalytic Psychology 12 (2), 247258. doi.org/10.1037/h0079625.

[23] Spoormaker, V. I., Schredl, M., and Van den Bout, J. (2006). Nightmares: from anxiety symptom to sleep disorder. Sleep Medicine Reviews, 10, 19-31. doi: 10.1016/j.smrv.2005.06.001.

[24] Bagby, R. M., Taylor, G. J., Parker, J. D. A., and Dickens, S. E., (2006). The development of the Toronto Structured Interview for Alexithymia: Item selection, factor structure, reliability and concurrent validity. Psychotherapy and Psychosomatics, 75, 25-39. doi.org/10.1159/000089224.

[25] Caretti, V., Porcelli, P., Solano, L., Schimmenti, A., Bagby, R. M., and Taylor, G. J. (2011). Reliability and validity of the Toronto Structured Interview for Alexithymia in a mixed clinical and nonclinical sample from Italy. Psychiatry $\begin{array}{llll}\text { Research, } & 187 & \text { (3), } & \text { 432-436. }\end{array}$ 10.1016/j.psychres.2011.02.015.

[26] Inslegers, R., Löbel, S., Dittrich, D., Bagby, R. M., Taylor, G. J., Quilty, L. C., Spitzer, C., Barnow, S., Mathier, F., Jenewein, J., Freyberger, H. J., Rufer, M. (2013). The Dutch language version of the Toronto Structured Interview for Alexithymia: reliability, factor structure and concurrent validity. Psychologica Belgica, 53 (1), 93-116. doi.org/10.5934/pb-53$1-93$.

[27] Montebarocci, O., and Surcinelli, P. (2018). Correlations between TSIA and TAS-20 and their relation to self-reported negative affect: a study using a multi-method approach in the assessment of alexithymia in a nonclinical sample from Italy. Psychiatry Research, 270, 187-193. doi: org/10.1016/j.psychres.2018.09.036.

[28] Taylor, G. J., and Bagby, R. M. 2013. Psychoanalysis and empirical research: the example of alexithymia. Journal of the American Psychiatric Association 61: 99-133. doi. $10.1177 / 0003065112474066$.

[29] Tibon, S., Weinberger, Y., Handelzaltz, J. E., and Porcelli, P. (2005). Construct validation of the Rorschach reality-fantasy scale in alexithymia. Psychoanalytic Psychology, 22, 508-523. doi.org/10.1037/07369735.22.4.508.

[30] Sekely, A., Taylor, G. J., and Bagby, R. M., (2018). Developing a short version of the Toronto Structured Interview for Alexithymia using item response theory. Psychiatry Research, 266, 218-227. doi: 10.1016/j.psychres.2018.03.002.

[31] Schredl, M. (2002). Questionnaires and diaries as research instruments in dream research: methodological issues. Dreaming, 12, 17-26.

[32] Schredl, M., Berres, S., Klingauf, A., Schellhaas, S., and Göritz, A. (2014). The Mannheim Dream questionnaire (MADRE): Retest reliability, age and gender effects. International Journal of Dream Research, 7 (2). 141-147. doi.org/10.11588/ijodr.2014.2.16675.

[33] Pedrabissi, L., and Santinello, M., Verifica della validità dello STAI forma Y di Spielberger [Verification of the validity of the STAI, Form Y, by Spielberger], (1989), Giunti Organizzazioni Speciali.

[34] Spielberger, C. D., "State-Trait Anxiety Inventory: Bibliography (2nd ed.) ", 1989, Palo Alto, CA: Consulting Psychologists Press. 
[35] Beck, A. T., Steer, R. A., and Brown, G. K., "Manual for the Beck Depression Inventory-II", 1996, San Antonio, TX: Psychological Corporation.

[36] Ghisi, M., Flebus, G. B., Montano, A., Sanavio, E., and Sica, C., "Beck Depression Inventory - II (BDI-II) Manuale", 2006, Firenze: O. S. Organizzazioni Speciali.

[37] Godin, I., Montplaisir, J., Gagnon, J. F., and Nielsen, T. (2013). Alexithymia associated with nightmare distress in idiopathic REM sleep behavior disorder. Sleep, 36 (12), 19571962.

[38] American Academy of Sleep Medicine, "The international classification of sleep disorders. (ICSD-3) ", 2014, Darien, IL: AASM.

[39] Schredl, M., and Erlacher, D. 2004. Lucid dreaming frequency and personality. Personality and Individual Differences, 37, 1463-1473.

[40] Funkhouser, A. T. 2009. Survey data, http://www.dejaexperience-research.org.

[41] Neppe, V. M., “The Psychology of Déjà Vu: Have I Been Here Before?”, 1983, Witwatersrand University Press.

[42] Clark, L. A., and Watson D. (1995). Constructing validity: basic issues in objective scale development. Psychological Assessment, 7, 309-319.

[43] Netemeyer, R. G., Bearden, W. O., and Sharma, S., "Scaling Procedures: Issues and Applications", 2004, Thousand Oaks, Sage Publications.

[44] Cortina, J. M. (1993). What is coefficient alpha? An examination of theory and applications. Journal of Applied Psychology, 78, 98-104.

[45] Fleiss, J. L., and Shrout P. E. (1977). The effects of measurement errors on some multivariate procedures. American Journal of Public Health 67, 1188-1191.
[46] Landis J. R., and Koch G. G. 1977. The measurement of observer agreement for categorical data. Biometrics 33, 159174.

[47] Bernstein, D. M., and Belicki, K. (1995). On the psychometric properties of retrospective dream content questionnaires. Imagination, Cognition and Personality, 15, 351-364.

[48] Zadra, A., Pilon, M., and Donderi, D. C. (2006). Variety and intensity of emotions in bad dreams and nightmares. Journal of nervous and mental disease, 194, 249-254. Canadian Psychology, 34 (2a), 294.

[49] Sno, H. N., Linszen, D. H., and De Jonghe, F. (1992b). Empirical research on déjà vu experiences: a review. Behavioral Neurology, 5, 151-161.

[50] Sno, H. N., and Draaisma, D. (1993). An early Dutch study of déjà vu experiences. Psychological Medicine, 23 (1): 17-26, 1993, Doi: 10.1017/S0033291700038812\}.

[51] Wolfradt, U. (2000). Zeitschrift für klinische Psychologie, Psychiatrie und Psychotherapie, 48 (4): 359-376.

[52] Arango-Muñoz, S. (2013b). Scaffolded memory and metacognitive feelings. Review of Philosophy and Psychology 4 (1): $135-152$.

[53] Lacinova, L., Michalcakova, R. N., Sirucek, J., Jezek, S., Chromec, J., Masopustova, Z., Urbanek, T., Brazdil, M. (2016). Deja Vu Experiences in Healthy Czech Adults. Journal of Nervous and Mental disease, 204 (12), 925-930. doi: 10.1097/NMD.0000000000000573.

[54] Funkhouser, A., and Schredl, M. (2010). The frequency of déjà vu (déjà rêve) and the effects of age, dream recall frequency and personality factors. International Journal of Dream Research 3/1, 60-64.

[55] Zunker, M., Althoff, H. K., Apel, J., Lässig, H. S., Schültke, L., and Schredl, M. (2015). Comparing questionnaire and diary measures for eliciting nightmare frequency. International Journal of Dream Research, 8/2, 129-134. 